Published as: Vanclooster, A., De Maeyer, P., Fack, V., \& Van de Weghe, N. (2013). Calculating

least risk paths in 3D indoor space. Paper presented at the 8th 3D GeoInfo conference, Istanbul,

Turkey, 27-29 November 2013.

\title{
calculating least risk paths in 3D indoor space
}

\author{
A. Vanclooster ${ }^{\mathrm{a}, *}$, Ph. De Maeyer ${ }^{\mathrm{a}}$, V. Fack ${ }^{\mathrm{b}}$, N. Van de Weghe ${ }^{\mathrm{a}}$ \\ Ghent University, Krijgslaan 281, B-9000 Ghent, Belgium \\ ${ }^{a}$ Dept. of Geography - (Ann.Vanclooster, Nico.VandeWeghe, Philippe.DeMaeyer)@UGent.be \\ ${ }^{\mathrm{b}}$ Dept. of Applied Mathematics and Computer Science - Veerle.Fack@UGent.be
}

KEY WORDS: Indoor navigation, 3D algorithms, cognitive wayfinding

\begin{abstract}
:
Over the last couple of years, research on indoor environments has gained a fresh impetus; more specifically applications that support navigation and wayfinding have become one of the booming industries. Indoor navigation research currently covers the technological aspect of indoor positioning and the modelling of indoor space. The algorithmic development to support navigation has so far been left mostly untouched, as most applications mainly rely on adapting Dijkstra's shortest path algorithm to an indoor network. However, alternative algorithms for outdoor navigation have been proposed adding a more cognitive notion to the calculated paths and as such adhering to the natural wayfinding behaviour (e.g. simplest paths, least risk paths). These algorithms are currently restricted to outdoor applications. The need for indoor cognitive algorithms is highlighted by a more challenged navigation and orientation due to the specific indoor structure (e.g. fragmentation, less visibility, confined areas...). As such, the clarity and easiness of route instructions is of paramount importance when distributing indoor routes. A shortest or fastest path indoors not necessarily aligns with the cognitive mapping of the building. Therefore, the aim of this research is to extend those richer cognitive algorithms to three-dimensional indoor environments. More specifically for this paper, we will focus on the application of the least risk path algorithm of Grum (2005) to an indoor space. The algorithm as proposed by Grum (2005) is duplicated and tested in a complex multi-storey building. The results of several least risk path calculations are compared to the shortest paths in indoor environments in terms of total length, improvement in route description complexity and number of turns. Several scenarios are tested in this comparison: paths covering a single floor, paths crossing several building wings and/or floors. Adjustments to the algorithm are proposed to be more aligned to the specific structure of indoor environments (e.g. no turn restrictions, restricted usage of rooms, vertical movement) and common wayfinding strategies indoors. In a later stage, other cognitive algorithms will be implemented and tested in both an indoor and combined indoor-outdoor setting, in an effort to improve the overall user experience during navigation in indoor environments.
\end{abstract}

\section{INTRODUCTION AND PROBLEM STATEMENT}

Over the last decade, indoor spaces have become more and more prevalent as research topic within geospatial research environments (Worboys, 2011). Past developments in the modelling and analysis of three-dimensional environments have already given us a better structural understanding of the use and possibilities of indoor environments (Becker et al., 2013; Boguslawski et al., 2011). These evolutions combined with the rapid progress in spatial information services and computing technology ( $\mathrm{Li}$ and Lee, 2010) have put three-dimensional modelling and analyses more and more in the spotlight. Also, given the fact that as human beings we spend most of our time indoors (Jenkins et al., 1992), indoor environments have become an indispensable part of current geospatial research.

Within indoor research, applications that support navigation and wayfinding are of major interest. A recent boost in technological advancements for tracking people in indoor environments has led to increasing possibilities for the development of indoor navigational models (Mautz et al. 2010). Alternatively, several researchers have developed a wide variety of indoor navigational models ranging from abstract space models (Becker et al. 2009) and 3D models (Coors 2003, Li \& $\mathrm{He} 2008$ ) to pure network models (Jensen et al. 2009, Karas et al. 2006, Lee 2001, Lee 2004). While these models might be useful in specific situations, a general framework for indoor navigation modelling has still to reach full maturity (Nagel et al. 2010). Far more recent is the commercial interest with public data gathering for navigation support in several indoor

* Corresponding author. This is useful to know for communication with the appropriate person in cases with more than one author. 
buildings (e.g. Google Maps Indoor), which demonstrates the current importance of this application field.

While a considerate amount of work is oriented to the abstract modelling and technological aspect of navigation, the algorithmic development to support navigation in indoor built environments has so far been left mostly untouched. Appropriate and accurate algorithmic support is nonetheless a necessary component for a successful wayfinding experience. In outdoor research, a wide variety of different algorithms exist, initially originating from shortest path algorithms, studied for over 50 years in mathematical sciences (Cherkassky et al. 1996). Many of them are based on the famous Dijkstra shortest path algorithm (Dijkstra 1959) with gradually more and more adaptations and extensions for better performance in terms of speed, storage and calculation flexibility (Zhan and Noon 1998). Over time, alternative algorithms were proposed adding a more cognitive notion to the calculated paths and as such adhering to the natural wayfinding behavior in outdoor environments. Examples are hierarchical paths (Fu et al. 2006), paths minimizing route complexity (Duckham and Kulik 2003, Richter and Duckham 2008) or optimizing risks along the described routes (Grum, 2005). The major advantage of those algorithms is their more qualitative description of routes and their changed embedded cost function, simplifying the use and understanding of the calculated routes and as such improving the entire act of navigation and wayfinding.

Algorithms for 3D indoor navigation are currently restricted to Dijkstra or derived algorithms. To date, only few researchers have attempted to approach algorithms for indoor navigation differently, for example incorporating dynamic events (Musliman et al., 2008), or modelling evacuation situations (Atila et al., 2013; Vanclooster et al., 2012). However, the need for more cognitively rich algorithms is even more pronounced in indoor space than outdoors. This has its origin in the explicit distinctiveness in structure, constraints and usage between indoor and outdoor environments. Outdoor environments are commonly described as continuous with little constraints, while the perception of buildings is strongly influenced by the architectural enclosures (Li, 2008; Walton and Worboys, 2009). Also, wayfinding tasks in multi-level buildings have proven to be more challenging than outdoors, for reasons of disorientation (due to multiple floor levels and staircases), and less visual aid (e.g. landmarks are less obviously recognizable; corners and narrow corridors prevent a complete overview) (Hölscher et al., 2007). As such, building occupants are faced with a deficient perspective on the building structure, influencing their movement behaviour (Hölscher et al., 2007). Algorithms developed to support a smooth navigation will have to consider these intricacies. Existing shortest or fastest paths are not necessarily the easiest for people to understand and risks of getting lost are greater than using appropriate algorithms. As such, route instructions should be more carefully designed to align with the human cognitive mapping of indoor spaces.

The main goal of this paper is to translate existing outdoor cognitive algorithms to an indoor environment and compare their efficiency and results in terms of correctness, difference to common shortest path algorithms and their equivalents in outdoor space. Based on the results of this implementation, a suggestions for a new and improved algorithm will be stated, which is more aligned to the specific context of indoor environments and wayfinding strategies of users indoor. In this paper, we currently focus on the implementation and adjustment of the least risk path algorithm as described by Grum (2005).
The remainder of the paper is organized as follows. Section 2 elaborates on the definition of the least risk path algorithm and its relationship to other cognitive algorithms and the shortest path algorithm. In section 3, the indoor dataset is presented in combination with the choices and assumptions made when developing the indoor network model. In the case study in section 4, the outdoor least risk path algorithm is duplicated and implemented in an indoor setting with multiple analyses comparing its results. Section 5 discusses multiple improvements to be made to the original algorithm to be more compatible with indoor networks. This paper is completed with a conclusion on the discussed issues.

\section{LEAST RISK ALGORITHM}

The ultimate goal of cognitive algorithms is to lower the cognitive load during the wayfinding experience. Various cognitive studies have indicated that the form and complexity of route instructions is equally important as the total length of path for humans when navigating (Duckham and Kulik, 2003). This is the reason why several algorithms have been developed for outdoor space with the purpose of simplifying the navigation task for unfamiliar users. In this paper we specifically focus on the least risk algorithm (Grum, 2005) and its implementation in a three-dimensional building. More specifically, we want to investigate whether or not the least risk path has the same connotation and importance in indoor spaces as in outdoor space where it was developed.

The least risk path as described by Grum (2005) calculates the path between two points where a wayfinder has the least risk of getting lost along the path. The risk of getting lost is measured at every intersection with the cost of the risk calculated as the cost for taking the wrong decision at the intersection. This algorithm assumes that the person taking the path is unfamiliar with its environment (and as such local landmarks). Also, when taking a wrong path segment, the wayfinder notices this immediately and turns back at the next intersection (Grum, 2005). While the algorithm assumes that an unfamiliar user immediately notices a wrong choice, the author also acknowledges that the algorithm needs to be tested for its representativeness of the actual behavior of users.

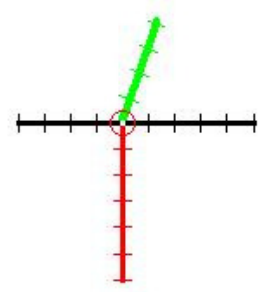

Figure 1: Intersection with red line the way the wayfinder came from, green line the way the wayfinder should go and black lines the wrong choices (Grum, 2005).

The formula for the calculation of the risk value at a certain intersection and the total risk of an entire path $\mathrm{p}$ is as follows:

Risk_Value $(i)=\frac{2 * \sum \text { length_wrong_choices }}{\text { possible_choices }}$

Total_Risk $(p)=\sum$ risk_values $(i)+\sum$ lengths 
From the formulas it appears that the risk value is dependent on the number of street segments converging on the intersection, combined with the length of each individual segment. The risk value of an intersection increases with more extensive intersections and with many long edges that could be taken wrongly. The algorithm favours paths with combined long edges and easy intersections. Applied to indoor environments, it could be assumed that the least risk path might be quite similar to the shortest path and simplest path. Indoor spaces often consist of many decision points and short edges, along long corridors making derivations of the shortest path more difficult than outdoors. This will be examined in the following sections.

The algorithmic structure of the least risk path algorithm is similar to Dijkstra with a continuous loop over all nodes including three consecutive steps:

1. Detect the next smallest node

2. Change the selected node to the next smallest node

3. Adjust the cost values for adjacent nodes

It is only in the third step that the least risk path differs from the Dijkstra algorithm since the cost value is not only based on the length of the edge but also on the risk value of each intersection that is passed. This risk value is dependent on the previous route taken to reach the selected node and the length of its adjacent edges. The following steps in the 'adjust cost section' are consecutively executed:

1. Calculate the number of edges leaving from selected node and select each edge successively

2. Two options:

2a. Endnode of selected edge has not been selected:

- Calculate possible total risk values for endnode based on all possible routes arriving in selected node

- Store the minimal value by comparing it with the currently stored value in endnode

2b. Endnode of selected edge has been selected BUT adjacent nodes have not been selected:

- Calculate the number of edges leaving from endnode and select each edge successively

- Calculate total risk values for endnode based on all possible routes arriving in selected node and the connection between the selected node and its adjacent node

- Store the minimal value by comparing it with the currently stored value

The example below shows that starting in the selected node, first nodes $\mathrm{N}_{1}$ en $\mathrm{N}_{2}$ will be checked. $\mathrm{N}_{1}$ has not yet been selected (option a) and will be calculated as a path coming from selected node and its consecutive parent node. Node $\mathrm{N}_{2}$ has already been calculated but one of its adjacent nodes has not. Therefore node $\mathrm{N}_{22}$ could possibly have a shorter path coming from the selected node and this will be checked through option $\mathrm{b}$ of the algorithm.

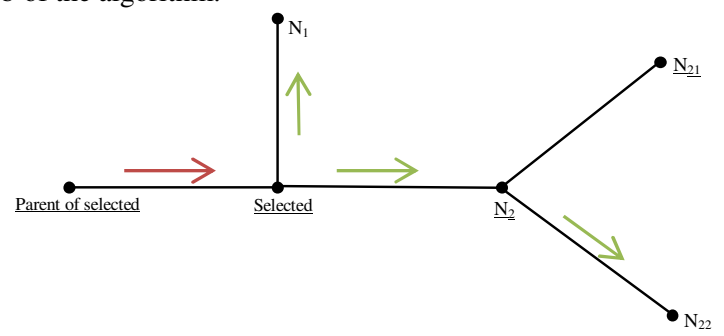

Figure 2: Example of the implementation of the least risk path algorithm (The underlined nodes have already been selected. Nodes $\mathrm{N}_{1}$ and $\mathrm{N}_{22}$ will be calculated starting in the selected node).
For each path, the total length and risk values for the intermediate nodes are calculated in both the shortest path and least risk path algorithm.

Given the fact that the only difference with the Dijkstra algorithm is in the cost calculation, and there the additional calculations only affect the amount of edges in the selected node, the computational complexity is similar to Dijkstra, being $\mathrm{O}\left(\mathrm{n}^{2}\right)$.

\section{INDOOR DATASET}

The algorithms developed require to be thoroughly tested in an extensive and complex indoor environment to be a valid alternative for outdoor algorithmic testing. Although the authors realize that using a single specific building dataset for testing can still be too limited to generalize the obtained results, we tried to map a building with several features that are quite common for many indoor environments. The dataset for our tests consist of the 'Plateau-Rozier' building of Ghent University (Fig. 3). It is a complex multistory building where several wings and sections have different floor levels and are not immediately accessible. It is assumed that the mapped indoor space is complex enough with many corners and decision points to assume reasonable wayfinding needs for unfamiliar users. Previous research executed in this building has shown that even familiar users have considerate difficulty recreating a previously shown route through the building (Viaene and De Mayer, 2013).

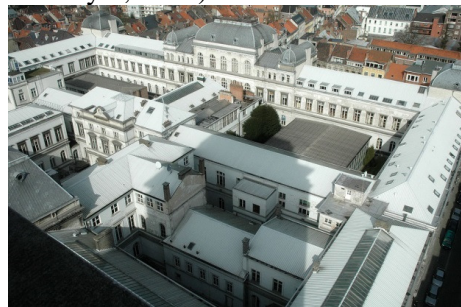

Figure 3: View of the 'Plateau-Rozier' building of Ghent University (Source: http://www.gentblogt.be/2006/11/22/hetwitte-gebouw)

The dataset is based on CAD floor plans which are transformed to ArcGIS shapefiles for additional editing and querying. For application of the least risk and shortest path algorithm, the original floor plans have to be converted into a threedimensional indoor network structure. Automatic derivation of indoor networks has long been focused on as one of the problematic areas for indoor navigation applications. Recent efforts have shown possibilities of automatically assigning nodes to each room object and connecting them when they are connected in reality (Anagnostopoulos et al., 2005; Meijers et al., 2005; Stoffel et al., 2008). However, the development of a comprehensive methodology for automatic network creation requires a thorough foundation and agreement on the appropriate and optimal (i.e. user friendly) network structure of indoor environments which supports the user in his navigation task (Becker et al., 2009). Therefore, in most existing indoor navigation applications, the data is still mostly manually transformed into graph structures. As such, we decided to manually create the network based on the subdivision into separate rooms. 


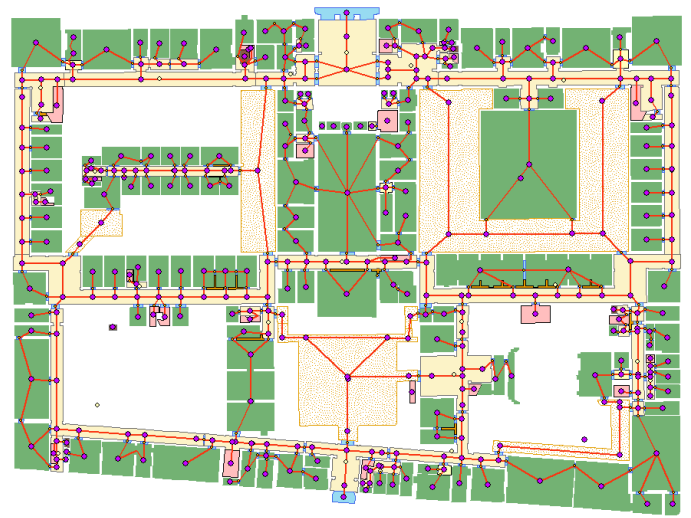

Figure 4: Floor plan of the ground floor with network visualization.

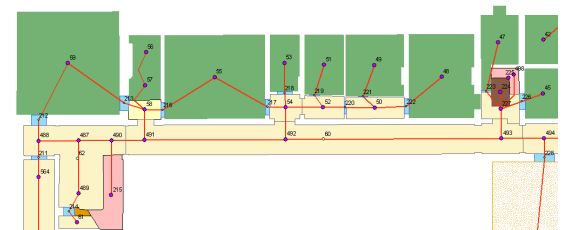

Figure 5: Detail of the ground floor's network.

The network structure is chosen to be compliant to Lee's Geometric Network Model (Lee, 2004) as this is one of the main accepted indoor data structures. In this model, each room is transformed into a node, forming a topologically sound connectivity model. Afterwards, this network is transformed into a geometric model by creating a subgraph for linear phenomena (e.g. corridors), as such enabling network analysis (Fig. 5). The position of the node within the room is chosen to be the geometrical center point of the polygons defining the rooms. This premise implies that the actual walking pattern will sometimes not be conform to the connectivity relationships in the network inducing small errors in the calculations of shortest and least risk paths (as shown in Fig. 5 for nodes 59 and 55). We will need to verify whether or not this error is significant in the total cost of certain paths. The selection of corridors to be transformed into linear features is based on the map text labels indicating corridor functionality. These areas also appear to be perceived as corridors when inspecting the building structure itself in the field. Obviously, this topic is depending on personal interpretation and choice. Therefore, in a future part of this research, the dependency of the performance of cognitive algorithms on the indoor network topology will be investigated.

\section{IMPLEMENTATION AND ANALYSIS OF LEAST RISK PATHS IN INDOOR SPACE}

\subsection{Analysis of least risk paths within indoor space}

4.1.1 Analysis of the entire dataset: The entire dataset consists of more than 600 nodes and more than 1300 edges which required a computation of almost 800.000 paths to exhaustively calculate all possible paths between all nodes for both the shortest as well as the least risk path algorithm.

As stated before, we would like to investigate whether least risk paths have a similar advantage to shortest paths in terms of navigational complexity as in outdoor space. Given the definition of least risk paths, we put forward the following hypotheses. First, the length of a path described by the least risk path algorithm is expected to be equal or longer than shortest path. As such, it provides a measure of the detour a wayfinder would need to take when using a path that is less difficult to get lost on. Second, the risk values of the shortest path will be equal or larger than for the least risk path. The least risk paths will more likely take routes with fewer intersections, away from the major corridors where many choices appear. It will also take longer edges while the shortest path will go for the most direct option ignoring the complexity of the individual intersections. Third, the total risk value for the shortest path will be equal or higher than for the least risk paths as this is the minimization criterion for the least risk algorithm. Above aspects are analyzed in the following paragraphs by comparing paths calculated by the least risk path algorithm and those calculated by the Dijkstra shortest path algorithm. These results aim to provide an indication of the balance struck by the different algorithms between the desire for direct routes and less risky routes.

\begin{tabular}{|l|rrr|}
\hline & \multicolumn{1}{|c}{$\begin{array}{c}\text { Total Cost } \\
\text { Difference }(\mathrm{m})\end{array}$} & $\begin{array}{c}\text { Length } \\
\text { Difference }(\mathrm{m})\end{array}$ & $\begin{array}{c}\text { Risk Value } \\
\text { Difference }(\mathrm{m})\end{array}$ \\
\hline Average & 11,11 & $-4,47$ & 15,59 \\
Min & 0,00 & $-74,63$ & 0,00 \\
Max & 135,48 & 0,00 & 145,73 \\
\hline
\end{tabular}

Figure 6: Summary of the entire dataset

On average, the difference in path length for least risk paths is around $4,5 \mathrm{~m}$ with a decrease in risk value of $15,5 \mathrm{~m}$. The values comparing the Dijkstra algorithm with the least risk path algorithm (total risk value minimization) align with the hypothesis stated before, with an increase in risk values for shortest paths and an increase in length values for least risk paths.

Over the entire dataset, a least risk path is on average $4 \%$ longer than its respective shortest path (using both the calculations of Duckham and Kulik (2003) as well as those from Jiang and Liu (2011)). Although 55\% of least risk paths are longer than the shortest paths, the majority (almost 99\%) of the paths are less than a quarter longer. A classification of the path differences between shortest and least risk paths is shown in Fig. 7.

\begin{tabular}{|l|r|r|}
\hline LengthIncrease & Nr of paths & \% of total paths \\
\hline Equal & 161613 & $46,74 \%$ \\
$\mathbf{5 \%}$ or $>$ & 96491 & $27,90 \%$ \\
$\mathbf{1 0 \%}$ or $>$ & 45718 & $13,22 \%$ \\
$\mathbf{2 5 \%}$ or $>$ & 4522 & $1,31 \%$ \\
$\mathbf{5 0 \%}$ or $>$ & 159 & $0,05 \%$ \\
\hline Total & 345785 & $100,00 \%$ \\
\hline
\end{tabular}

Figure 7: Classification of paths

The average path lengths of the shortest and least risk paths were almost equal $(109,22 \mathrm{~m}$ to $113,69 \mathrm{~m}$ with standard deviations of $45,89 \mathrm{~m}$ and $48,74 \mathrm{~m}$ respectively), intensifying the found limited differences on a whole between shortest and least risk paths in indoor spaces.

Figure 8 summarizes the entire data set of paths and its individual differences. More specifically, it visualizes the spatial distribution of the standard deviation for all least risk paths starting in that point. The standard deviations have been classified in five quintiles (five classes with equal cardinality), similar to Duckham and Kulik (2003) analysis. The figure shows generally low standard deviations (lighter yellow data points) on the first floor and in lesser connected areas of the building. The higher standard deviations (dark brown data points) generally occur on the ground floor in denser connected areas and around staircases both on the ground and first floor. This greater variability can be interpreted as a result of the 
deviations of the least risk path from the shortest path being more pronounced at the rooms with many options like around staircases where paths can be significantly different in the final route. Starting locations within isolated areas (e.g. on the first floor) have no option but to traverse similar areas to reach a staircase and deviate from there onwards.

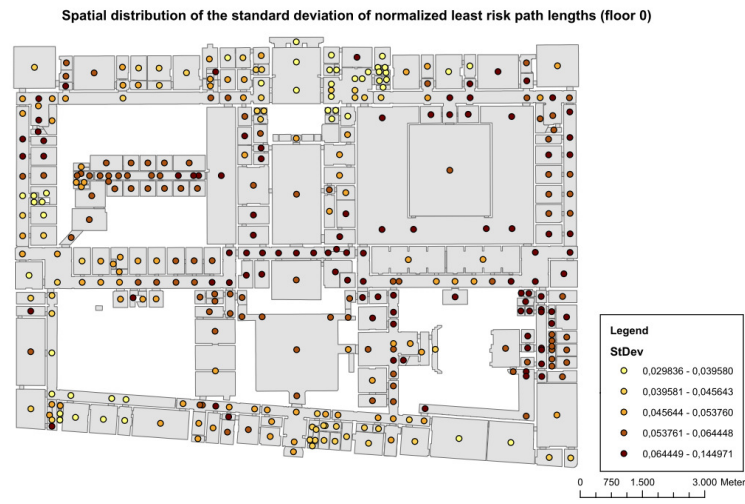

Spatial distribution of the standard deviation of normalized least risk path lengths (floor 1)
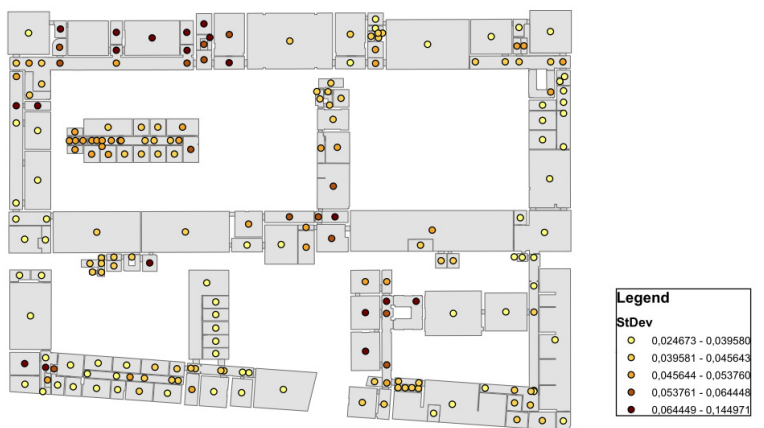

Figure 8: Spatial distribution of the standard deviation of normalized least risk path lengths

The ground floor standard deviations are generally larger due to a network with higher complexity and connectivity. This trend can also be detected in the classification of the paths and their respective increase in length by chosing a less risky road. $80 \%$ of the longest paths (compared to the shortest path) with an increase of $50 \%$ or more are found on the ground floor, while half of the paths on the first floor are equal to their respective shortest path.
4.1.2 Analysis of selected paths: In this section, a few example paths are highlighted for further analysis. In figure 9 an example shortest and least risk path is calculated and visualized. Both the starting and the end point are on the ground floor of the building. There is a significant visual difference in path choice of the example route. The values in figure 10 show an expected lower total risk value for the least risk path with a considerable lower risk value at the decision points. The least risk path is $43 \%$ longer than the shortest path, which minimizes its total length. The shortest path has 6 turns in its description, while the least risk path requires 7 turns. This example shows a 'worst-case scenario' as it has one of the biggest differences in total path length of the entire dataset. While the shortest path takes the direct route following main corridors, the least risk path avoids certain areas to prevent wayfinders from getting lost as easily. However, from this figure, it is not entirely visible why the least risk path deviates from the shortest path in favour of using its calculated route. It should be tested in the field if the route is actually easier and safer for wayfinding. However, the authors doubt that this would be the case. Figure 9 is an example of why the least risk path indoor might need to be differently implemented.

Visualization of a typical least risk and shortest path

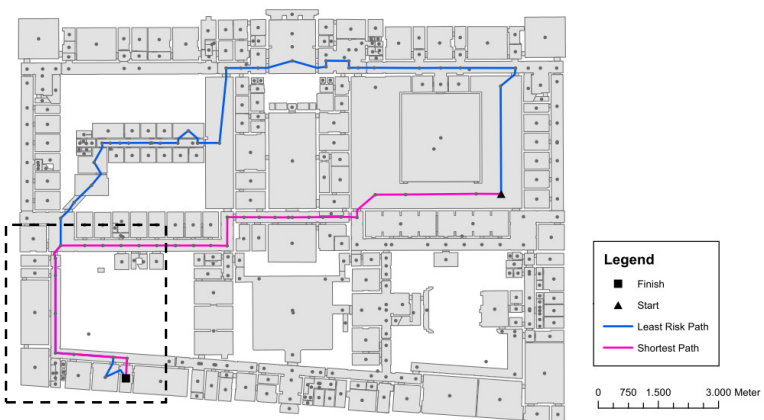

Figure 9: Comparison of a typical shortest and least risk path

\begin{tabular}{|l|r|r|r|r|}
\hline Path type & $\begin{array}{c}\text { Risk values of } \\
\text { decision points }(\mathbf{m})\end{array}$ & $\begin{array}{l}\text { Length of path } \\
\text { segments }(\mathbf{m})\end{array}$ & $\begin{array}{c}\text { Risk value of the } \\
\text { entire path }(\mathbf{m})\end{array}$ & $\begin{array}{c}\text { Number of } \\
\text { turns }\end{array}$ \\
\hline Shortest path & 274,27 & 170,80 & 445,07 & 6 \\
\hline Least risk path & 166,36 & 245,43 & 411,79 & 7 \\
\hline
\end{tabular}

Figure 10: Comparison of the risk values between an example shortest and least risk path

A comparison of the lengths of the least risk and shortest paths for one set of paths from a single source to every other vertex in the data set is shown in Fig. 11. The figure provides a scatter plot of the normalized least risk path length (the ratio of least risk to shortest path lengths), plotted against shortest path length. In this example, more than $98 \%$ of the least risk paths are less than $50 \%$ longer than the corresponding shortest path. 


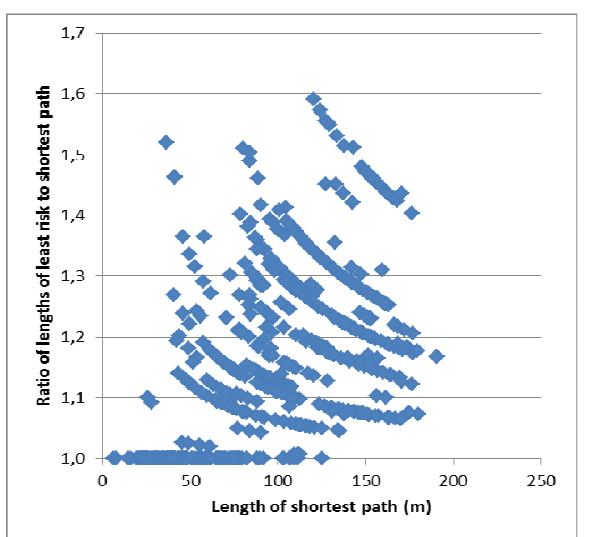

Figure 11: Graph of the ratio of least risk on shortest path length to the shortest path length

Most paths are (almost) similar in length to its shortest path equivalent. Often only a small change in path choice can be found with a difference of only a couple of nodes compared to the shortest path. On the other hand, the strongly correlated stripes going from top left to bottom right in the graph exhibit blocks of correlated paths with very similar path sequences throughout their entire route. These occur because many adjacent nodes are required to take similar edges to reach their destination. This can also be seen in Fig. 9. The nodes within the dashed rectangle all take the same route for both their least risk and shortest path, resulting in connected ratios in Fig. 11.

\subsection{Analysis of indoor least risk paths compared to the results in outdoor space}

In this section, several of the data obtained before will be compared with the results obtained by the calculations of least risk paths by Grum (2005) and simplest paths by Duckham and Kulik (2003). We mainly want to investigate whether we can draw the same conclusions from our results of the calculations in indoor space as those from outdoor space. Also, the question is raised if the size of the difference is equivalent to outdoors.

A comparison with the result obtained by Grum (2005) is difficult as the author only calculated a single path in outdoor space. In both cases, the total risk value for the least risk path is minimal and the length is longer than its shortest path. The outdoor least risk path is $9 \%$ longer than the shortest path, while in our dataset an average increase of $4 \%$ is detected. However, the number of turns in our example path (Fig. 10) is higher for the least risk path compared to the shortest path. Other paths in our dataset have less turns than their shortest path equivalent. This does not seem to match with the results from the outdoor variant. An explanation could be that the author only works with a limited outdoor dataset. Also, the least risk path indoor might have a different connotation because of the description of the indoor network. Due to the transformation of the corridor nodes to a linear feature with projections for each door opening, the network complexity is equivalent to a dense urban network. However, the perception for an indoor wayfinder is totally different. While in outdoor space each intersection represents a decision point; in buildings, the presence of door openings to rooms on the side of a corridor is not necessarily perceived as single intersection where a choice has to be made. Often these long corridors are traversed as if it were a single long edge in the network.
Simplest paths have similarly to least risk paths the idea of simplifying the navigation task for people in unfamiliar environments. The cost function in both simplest and least risk paths accounts for structural differences of intersections, but not for functional aspects (direction ambiguity, landmarks in instructions...) like the simplest instructions algorithm (Richter and Duckham, 2008). However, the simplest path algorithm does not guarantee when taking one wrong decision that you will still easily reach your destination, while the least risk path tries to incorporate this while at the same time keeping the complexity of the instructions to a minimum. Several of the comparison calculations are similar to the ones calculated for simplest paths (Duckham and Kulik, 2003). At this point, we cannot compare actual values as it covers a different algorithmic calculation. In the future, we plan to implement the simplest path algorithm also in indoor spaces. However, it might be useful at this point to compare general trends obtained in both.

With regard to the variability of the standard deviations (Fig. 8) similar conclusions can be drawn. At the transition between denser network areas and more sparse regions, the variability tends to increase as a more diverse set of paths can be calculated. The sparse and very dense areas have similar ratios showing similar network options and path calculations.

The worst-case example can also be compared to a worst-case dataset of the outdoor simplest path. A similar trend in 'stripes' as found in the graph in Fig. 11 is also found in the outdoor simplest path results, also due to sequences of paths that are equal for many adjacent nodes.

\section{RECOMMENDATIONS FOR ADJUSTING THE LEAST RISK PATH ALGORITHM}

As the least risk paths in indoor space show not necessarily a lowered risk of getting lost, several adjustments to the algorithm will be proposed. These will be tested in future research as to result in a more cognitively accurate algorithm for wayfinding in indoor spaces.

Currently, the risk value of a decision point is calculated based on the assumption that the wayfinder recognizes his mistake at the first adjacent node and returns from there to the previous node. A question could be raised whether it is actually realistic that people already notice at the first intersection that they have been going wrong. An increasing compounding function could be suggested taking into account the possibility of going further in the wrong direction. Also, depending on the environmental characteristics, the chances of noticing a wrong decision can change dramatically. Research shows that landmarks for wayfinding are much harder to distinguish indoors than outdoors (Millonig et al., 2007). Additionally, the fact that you have to walk up and down staircases could be naturally having a greater weight because taking a wrong decision might result in walking up and down the stairs twice. On the other, chances of taking a wrong decision by changing floors are likely to be slimmer given the effort for vertical movement and a changed cognitive thinking.

In line with this last point, wayfinding research (Hölscher et al., 2009) showed the strategy choices people make when navigating in (un)familiar buildings. This research proves that people's strategy choice indoors varies with different navigation tasks. The main strategies for indoor wayfinding are recognized as central point strategy, direction strategy and floor strategy. Tasks with either a floor change or a building part change result in no problems, with the participants first changing to the 
correct floor or building part. However, for tasks with changes in both vertical and horizontal direction, additional information is required to disambiguate the path choice. An algorithm that wants to minimize the risk of getting lost in a building necessarily needs to account for these general indoor wayfinding strategies as they correspond to the natural way of multilevel building navigation for all types of participants.

In the current implementation of the least risk path algorithm, both the length of the path as well as the sum of the risk values at intermediate decision points have an equal weight in the calculation of the total risk value. Varying the individual weight of both parameters might results in a more cognitively correct calculation of the indoor least risk paths. Also, a more sophisticated algorithm could select routes that preferentially use more important or higher classified edges.

As previously mentioned, the description of the indoor network has a large influence on the results of the least risk comparisons. The introductions of many dummy nodes in front of doors that are not perceived as intersections, introduces a complexity in the risk value calculation, which seems to heavily influence our results. Therefore, the second stage of this research will investigate the importance and size of this dependency of the performance of cognitive algorithms on the indoor network topology.

\section{CONCLUSIONS}

In this paper, the least risk path algorithm as developed by Grum (2005) in outdoor space is implemented and tested in an indoor environment. The results of the tests on an indoor dataset show an average increase in path length of $4 \%$ compared to the shortest paths. Also, the initial hypotheses with respect to the ratio of results have proven to be correct. However, it appears to be difficult to visually see and understand the actual improvement in risk when calculating the total risk. The least risk path often passes by a great amount of complex intersections with many short edges. These paths will likely not be perceived by the wayfinder as less risky compared to the shortest path. However, this should be tested in the field. Comparisons of our results to the outdoor variant are difficult due to limited data outdoor. However, a similar increase in length has been found.

Our main conclusions from the analysis suggest that improvements to the indoor variant of the least risk algorithm are required, given the complexity of the current least risk paths. Changes in the calculation of the risk value, together with a weighing of the parameters will be tested. Also, the influence of the network structure will be investigated in future research in a search for optimizing the algorithm to be more compliant to the cognitive notion of indoor wayfinding.

\section{REFERENCES}

Anagnostopoulos, C., Tsetsos, V., Kikiras, P., \& Hadjiefthymiades, S. P. (2005). OntoNav: A Semantic Indoor Navigation System. Paper presented at the 1st Workshop on Semantics in Mobile Environments (in conjunction with MDM 2005), Ayia Napa, Cyprus.

Atila, U., Karas, I., \& Rahman, A. (2013). A 3D-GIS Implementation for Realizing 3D Network Analysis and Routing Simulation for Evacuation Purpose. In J. Pouliot,
S. Daniel, F. Hubert \& A. Zamyadi (Eds.), Progress and New Trends in 3D Geoinformation Sciences (pp. 249-260): Springer Berlin Heidelberg.

Becker, T., Nagel, C., \& Kolbe, T. (2009). A Multilayered Space-Event Model for Navigation in Indoor Spaces. In J. Lee \& S. Zlatanova (Eds.), 3D Geo-Information Sciences (pp. 61-77). Berlin, Germany: Springer.

Becker, T., Nagel, C., \& Kolbe, T. (2013). Semantic 3D Modeling of Multi-Utility Networks in Cities for Analysis and 3D Visualization. In J. Pouliot, S. Daniel, F. Hubert \& A. Zamyadi (Eds.), Progress and New Trends in 3D Geoinformation Sciences (pp. 41-62): Springer Berlin Heidelberg.

Boguslawski, P., Gold, C. M., \& Ledoux, H. (2011). Modelling and analysing 3D buildings with a primal/dual data structure. ISPRS Journal of Photogrammetry and Remote Sensing, 66(2), 188-197.

Cherkassky, B., Goldberg, A., \& Radzik, T. (1996). Shortest paths algorithms: Theory and experimental evaluation. Mathematical Programming, 73(2), 129-174.

Coors, V. (2003). 3D-GIS in Networking Environments. Computers, Environment and Urban Systems, 27(4), 345357.

Dijkstra, E. W. (1959). A Note on Two Problems in Connexion with Graphs. Numerische Mathematik, 1(1), 269-271.

Duckham, M., \& Kulik, L. (2003). "Simplest" Paths: Automated Route Selection for Navigation. In W. Kuhn, M. Worboys \& S. Timpf (Eds.), Spatial Information Theory. Foundations of Geographic Information Science (Vol. 2825, pp. 169-185): Springer Berlin/Heidelberg.

Fu, L., Sun, D., \& Rilett, L. R. (2006). Heuristic shortest path algorithms for transportation applications: State of the art. Computers \& Operations Research, 33(11), 3324-3343.

Grum, E. (2005). Danger of getting lost: Optimize a path to minimize risk. Paper presented at the 10th International Conference on Information \& Communciation Technologies (ICT) in Urban Planning and patial Development and Impacts of ICT on Physcial Space, Vienna, Austria.

Hölscher, C., Büchner, S. J., Meilinger, T., \& Strube, G. (2007). Map use and wayfinding strategies in a multi-building ensemble. Paper presented at the 2006 international conference on Spatial Cognition V: reasoning, action, interaction, Bremen, Germany.

Jenkins, P. L., Phillips, T. J., Mulberg, E. J., \& Hui, S. P. (1992). Activity patterns of Californians: Use of and proximity to indoor pollutant sources. Atmospheric Environment. Part A. General Topics, 26(12), 2141-2148.

Jensen, C. S., Lu, H., \& Yang, B. (2009). Graph Model Based Indoor Tracking. Paper presented at the 2009 Tenth International Conference on Mobile Data Management: Systems, Services and Middleware.

Jiang, B., \& Liu, X. (2011). Computing the fewest-turn map directions based on the connectivity of natural roads. International Journal of Geographical Information Science, 25(7), 1069-1082.

Karas, I., Batuk, F., Akay, A., Baz, I. (2006). Automatically Extracting 3D Models and Network Analysis for Indoors. In A. Abdul-Rahman, Zlatanova, S., Coors, V. (Ed.), Innovation in 3D-Geo Information System (pp. 395-404). Berlin Heidelberg: Springer.

Lee, J. (2001). 3D Data Model for Representing Topological Relations in Urban Features. Retrieved from http://gis.esri.com/library/userconf/proc01/professional/pap ers/pap565/p565.htm 
Lee, J. (2004). A Spatial Access-Oriented Implementation of a 3-D GIS Topological Data Model for Urban Entities. Geoinformatica, 8(3), 237-264.

Li, K.-J. (2008). Indoor Space: A New Notion of Space. In M. Bertolotto, C. Ray \& X. Li (Eds.), Web and Wireless Geographical Information Systems (Vol. 5373, pp. 1-3): Springer Berlin/Heidelberg.

Li, K.-J., \& Lee, J. (2010). Indoor Spatial Awareness Initiative and Standard for Indoor Spatial Data.

Li, Y., He, Z. (2008). 3D Indoor Navigation: a Framework of Combining BIM with 3D GIS. Paper presented at the 44th ISOCARP Congress 2008, Dalian, China.

Mautz, R., Kunz, M., \& Ingensand, H. (2010). Abstract Volume of the 2010 International Conference on Indoor Positioning and Indoor Navigation. Zurich, Switzerland.

Meijers, M., Zlatanova, S., \& Pfeifer, N. (2005). 3D GeoInformation Indoors: Structuring for Evacuation. Paper presented at the Next generation 3D City Models, Bonn, Germany.

Millonig, A., \& Schechtner, K. (2007). Developing LandmarkBased Pedestrian-Navigation Systems. Intelligent Transportation Systems, IEEE Transactions on, 8(1), 43-49.

Musliman, I. A., Abdul-Rahman, A., \& Coors, V. (2008). Implementing 3D network analysis in 3D-GIS. Paper presented at the 21st ISPRS Congress Silk Road for Information from Imagery, Beijing, China.

Nagel, C., Becker, T., Kaden, R., Li, K.-J., Lee, J., \& Kolbe, T. H. (2010). Requirements and Space-Event Modeling for Indoor Navigation OGC Discussion Paper (pp. 54). OGC.

Pepijn, V., \& De Maeyer, P. (2013). Detecting landmarks for use in indoor wayfinding. Ghent University, Ghent.

Richter, K.-F., \& Duckham, M. (2008). Simplest Instructions: Finding Easy-to-Describe Routes for Navigation. In $\mathrm{T}$. Cova, H. Miller, K. Beard, A. Frank \& M. Goodchild (Eds.), Geographic Information Science (Vol. 5266, pp. 274-289): Springer Berlin/Heidelberg.

Stoffel, E. P., Schoder, K., \& Ohlbach, H. J. (2008). Applying Hierarchical Graphs to Pedestrian Indoor Navigation. Paper presented at the Proceedings of the 16th ACM SIGSPATIAL international conference on Advances in geographic information systems, Irvine, California.

Vanclooster, A., Neutens, T., Fack, V., Van de Weghe, N., \& De Maeyer, P. (2012). Measuring the exitability of buildings: A new perspective on indoor accessibility. Applied Geography, 34(0), 507-518.

Walton, L. A., \& Worboys, M. (2009). Indoor Spatial Theory. www.spatial.maine.edu/ISAmodel/documents/IST_ISA09.p df

Worboys, M. (2011). Modeling Indoor Space. Paper presented at the Third ACM SIGSPATIAL International Workshop on Indoor Spatial Awareness (ISA 2011), Chicago, IL.

Zhan, F. B., \& Noon, C. E. (1998). Shortest path algorithms: An evaluation using real road networks. Transportation Science, 32(1), 65-73.

\section{ACKNOWLEDGEMENTS}

Financial support from the Flanders Research Foundation (FWO-Vlaanderen) is gratefully acknowledged. 